Journal of Mathematics and Statistics 6 (1): 68-71, 2010

ISSN 1549-3644

(C) 2010 Science Publications

\title{
The Time-Dependent Mean and Variance of the Non-Stationary Markovian Infinite Server System
}

\author{
Peter M. Ellis \\ Department of Management, Utah State University, Logan, Utah, USA
}

\begin{abstract}
Problem statement: In many queuing situations the average arrival and service rates vary over time. In those situations a transient solution for the state probabilities and mean and variance must be obtained. Approach: The mean and the variance of a particular infinite server model will be obtained using the state differential-difference equations and the factorial moment generating function. The average arrival and service rates will be taken to be dependent on time. The individual customer interarrival times and service time are assumed to be exponentially distributed. This is known as the Markovian system. Results: The mean and variance of the system will be established as solutions to two sequential linear ordinary differential equations. A comparison is also made to a previously known result for the corresponding system with a finite number of servers. Conclusion: Simple closed-form equations for the mean and variance of the system are presented.
\end{abstract}

Key words: Queues, infinite server, mean, variance

\section{INTRODUCTION}

In a recent article in this journal, El-Sherbiny (2010) obtained the closed form state probabilities for the $\mathrm{M} / \mathrm{M} / \infty$ system. He used the factorial moment generating function and the underlying state differential-difference equations to obtain the individual state probabilities when the average arrival rate and average service rate are not constant, but vary over time. The approach also incorporated Bessel functions in the expressions for the individual transient state probabilities. This study extends the El-Sherbiny (2010) contribution by also developing equations for the mean and variance of the same system.

Queuing systems are classified as $*_{1} / *_{2} / \mathrm{S}$, where $*_{1}$ is the arrival discipline, $*_{2}$ is the service discipline and $S$ is the number of servers available in parallel. The case of $*_{1}$ and $*_{2}$ being $\mathrm{M}$ is the presence of the lack-ofmemory or Markov property. That is, interarrival times and service times follow the negative exponential probability distribution. The $\mathrm{M} / \mathrm{M} / \infty$ service system is an extension of the M/M/S queuing system wherein the number of servers is infinite. This means that a newly arrived customer can always get directly into service without having to wait in line.

Interested readers are referred to the above ElSherbiny (2010) article for an excellent set of references to the history of related research. The $\mathrm{M} / \mathrm{M} / \infty$ service system has received growing interest in recent years because the insistence upon constant arrival and service rates is overly restrictive in many real applications.

\section{MATERIALS AND METHODS}

The mean and variance of the $\mathrm{M} / \mathrm{M} / \infty$ system will be developed here. The starting point will be the state differential-difference equations, as was done by ElSherbiny (2010). The classical moment generating function $\mathrm{Z}(\mathrm{x}, \mathrm{t})$ will be applied to the differentialdifference equations, first to obtain the mean $\mathrm{L}(\mathrm{t})$, then the variance $V(t)$. The mean and variance emerge from the moment generating function derivation as nonhomogeneous ordinary differential equations and are solved sequentially.

The M/M/ $\infty$ system: Let the average customer arrival rate be $\lambda(t)$ and the average service rate be $\mu(t)$ at time $t$. Let the expected number of customers in the system be $\mathrm{L}(\mathrm{t})$ and the variance of the number of customers in the system be $\mathrm{V}(\mathrm{t})$ at time $\mathrm{t}$. The number of customers in the system at any moment is $n=0,1, \ldots$. The probability of $n$ customers being in the system at time $t$ is $P_{n}(t)$. The number of customers in the system is known as the state of the system.

The $\mathrm{M} / \mathrm{M} / \infty$ system does not involve customer waiting time. All customers proceed directly into service upon arrival. Examples of application include recreational facilities such as parks, running tracks and swimming pools. Large automobile parking lots that are sure to not totally fill up also could conform.

The state differential-difference equations for the $\mathrm{M} / \mathrm{M} / \infty$ system are given in El-Sherbiny (2010) study as: 


$$
\begin{aligned}
P_{n}^{\prime}(t)=- & (\lambda(t)+n \mu(t)) P_{n}(t)+\lambda(t) P_{n-1}(t)+ \\
& (n+1) \mu(t) P_{n+1}(t) 0 \leq n<\infty
\end{aligned}
$$

In (1), take $\mathrm{P}_{-1}(\mathrm{t})$ to be zero in the equation for $P_{o}{ }^{\prime}(t)$. When $\lambda(t)=\lambda$ and $\mu(t)=\mu$ are both constant over time, the steady-state solution to the system is easy to obtain. Set all $P_{n}{ }^{\prime}(t)=0$ and solve recursively to obtain $\mathrm{P}_{\mathrm{n}}$ as Poisson with parameter $\lambda / \mu$. In this circumstance, $\mathrm{L}(\mathrm{t})=\mathrm{V}(\mathrm{t})=\lambda / \mu$. If either the arrival rate or the service rate varies over time then the system will be nonstationary (transient) and the steady-state solution is not applicable.

The variance: It is formally true that $\mathrm{L}(\mathrm{t})$ and $\mathrm{V}(\mathrm{t})$ are defined as:

$$
\begin{gathered}
\mathrm{L}(\mathrm{t})=\sum_{\mathrm{n}=0}^{\infty} n \mathrm{PP}_{\mathrm{n}}(\mathrm{t}) \\
\mathrm{V}(\mathrm{t})=\sum_{\mathrm{n}=0}^{\infty}(\mathrm{n}-\mathrm{L}(\mathrm{t}))^{2} \mathrm{P}_{\mathrm{n}}(\mathrm{t})
\end{gathered}
$$

Through completing the squares and summing it is also easily seen that:

$$
\sum_{n=0}^{\infty} n^{2} P_{n}(t)=V(t)+L^{2}(t)
$$

That substitution will be used several times here. We will obtain non-homogeneous first-order ordinary linear differential equations for $\mathrm{L}(\mathrm{t})$ and $\mathrm{V}(\mathrm{t})$. The two ordinary differential equations are solved sequentially.

Theorem: The variance of the distribution of state probabilities in the non-stationary $\mathrm{M} / \mathrm{M} / \infty$ system is given as:

$$
\begin{aligned}
\mathrm{V}(\mathrm{t})= & \mathrm{L}_{\mathrm{o}} \exp \left(-\int_{0}^{\mathrm{t}} \mu(\mathrm{s}) \mathrm{ds}\right)+ \\
& \left(\exp \left(-\int_{0}^{\mathrm{t}} \mu(\mathrm{s}) \mathrm{ds}\right) \int_{0}^{\mathrm{t}} \lambda(\mathrm{s})\left(\exp \int_{0}^{\mathrm{s}} \mu(\mathrm{r}) \mathrm{dr}\right) \mathrm{ds}\right) \\
& +\left(\mathrm{V}_{\mathrm{o}}-\mathrm{L}_{\mathrm{o}}\right) \exp \left(-2 \int_{0}^{\mathrm{t}} \mu(\mathrm{s}) \mathrm{ds}\right)
\end{aligned}
$$

Proof: The proof proceeds from the state differentialdifference Equations of (1) and the factorial moment generating function, as done by El-Sherbiny (2010). The factorial moment generating function $\mathrm{Z}(\mathrm{x}, \mathrm{t})$ is established as:

$$
\mathrm{Z}(\mathrm{x}, \mathrm{t})=\sum_{\mathrm{n}=0}^{\infty} \mathrm{x}^{\mathrm{n}} \mathrm{P}_{\mathrm{n}}(\mathrm{t})
$$

Then:

$$
\left.\frac{\partial Z(x, t)}{\partial x}\right|_{x=1}=\sum_{n=0}^{\infty} n x^{n-1} P_{n}(t)=L(t)
$$

Also:

$$
\begin{aligned}
& \frac{\partial^{2} Z(x, t)}{\partial x^{2} x=1} \sum_{n=0}^{\infty} n(n-1) x^{n-2} P_{n}(t) \\
& =V(t)+L^{2}(t)-L(t)
\end{aligned}
$$

Multiplying the state differential-difference Equations of (1) by $\mathrm{x}^{\mathrm{n}}$ and summing yields:

$$
\begin{aligned}
\frac{\partial Z(x, t)}{\partial t}= & -\lambda(t) \sum_{n=0}^{\infty} x^{n} P_{n}(t)-\mu(t) \sum_{n=0}^{\infty} n x^{n} P_{n}(t) \\
& +\lambda(t) \sum_{n=0}^{\infty} x^{n} P_{n-1}(t)+\mu(t) \\
& \sum_{n=0}^{\infty} x^{n}(n+1) P_{n+1}(t)
\end{aligned}
$$

Then:

$$
\begin{aligned}
\frac{\partial^{2} Z(x, t)}{\partial x \partial t}= & \sum_{n=0}^{\infty} n x^{n-1} P_{n}{ }^{\prime}(t) \\
= & -\lambda(t) \sum_{n=0}^{\infty} n x^{n-1} P_{n}(t)+\mu(t) \\
& \sum_{n=0}^{\infty} n^{2} x^{n-1} P_{n}(t)+\lambda(t) \\
& \sum_{n=0}^{\infty} n x^{n-1} P_{n-1}(t)+\mu(t) \\
& \sum_{n=0}^{\infty} n(n+1) x^{n-1} P_{n+1}(t)
\end{aligned}
$$

When $\mathrm{x}=1$, this becomes:

$$
\begin{aligned}
\frac{\partial^{2} Z(x, t)}{\partial x \partial t}= & -\lambda(t) L(t)-\mu(t)\left(V(t)+L^{2}(t)\right) \\
& +\lambda(t) L(t)+\lambda(t)+\mu(t) \\
& \sum_{n=0}^{\infty}((n+1)-1)(n+1) P_{n+1}(t)
\end{aligned}
$$

And since:

$$
\left.\frac{\partial^{2}}{\partial x \partial t} Z(x, t)\right|_{x=1}=L^{\prime}(t)
$$

We get:

$L^{\prime}(t)=-\mu(t) L(t)+\lambda(t)$ 
Equation 3 is a first-order non-homogeneous ordinary differential equation. Its solution is:

$$
\begin{aligned}
L(t)= & L_{0} \exp \left(-\int_{0}^{t} \mu(s) d s\right)+ \\
& {\left[\operatorname { e x p } ( - \int _ { 0 } ^ { t } \mu ( s ) d s ) \left(\int_{0}^{t} \lambda(s)(\exp \right.\right.} \\
& \left.\left.\left(\int_{0}^{s} \mu(r) d r\right)\right) d s\right]
\end{aligned}
$$

The partial derivative of (2) with respect to $\mathrm{x}$ gives:

$$
\begin{aligned}
\frac{\partial^{3} Z(x, t)}{\partial x^{2} \partial t}= & \sum_{n=0}^{\infty} n(n-1) x^{n-2} P_{n}{ }^{\prime}(t) \\
= & -\lambda(t) \sum_{n=0}^{\infty} n(n-1) x^{n-2} P_{n}(t)-\mu(t) \\
& \sum_{n=0}^{\infty} n^{2}(n-1) x^{n-2} P_{n}(t)+\lambda(t) \\
& \sum_{n=0}^{\infty} n(n-1) x^{n-2} P_{n-1}(t)+\mu(t) \\
& \sum_{n=0}^{\infty}(n+1)(n)(n-1) P_{n+1}(t)
\end{aligned}
$$

When this is evaluated at $x=1$ we get:

$$
\begin{aligned}
\frac{\partial^{3} Z(x, t)}{\partial x^{2} \partial t}= & \sum_{n=0}^{\infty} n(n-1) P_{n}{ }^{\prime}(t) \\
& =-\lambda(t) \sum_{n=0}^{\infty} n(n-1) P_{n}(t)-\mu(t) \\
& \sum_{n=0}^{\infty} n^{3} P_{n}(t)+\mu(t) \\
& \sum_{n=0}^{\infty} n^{2} P_{n}(t)+\lambda(t) \\
& \sum_{n=0}^{\infty}(n-1)^{2} P_{n-1}(t)+\lambda(t) \\
& \sum_{n=0}^{\infty}(n-1) P_{n-1}(t)+\mu(t) \\
& \sum_{n=0}^{\infty}(n+1)((n+1)-1) \\
& ((n+1)-2) P_{n+1}(t) \\
= & \lambda(t) L(t)-\mu(t) \sum_{n=0}^{\infty} n^{3} P_{n}(t) \\
& +\mu(t)\left[V(t)+L^{2}(t)\right]+\lambda(t) L(t)+\mu(t) \\
& \sum_{n=0}^{\infty}(n+1)((n+1)-1)((n+1)-2) P_{n+1}(t) \\
= & \lambda(t) L(t)+\mu(t)\left[V(t)+L^{2}(t)\right]+\lambda(t) L(t) \\
& -3 \mu(t)\left[V(t)+L^{2}(t)\right]+2 \mu(t) L(t)
\end{aligned}
$$

Since:

$$
\left.\frac{\partial^{2}}{\partial x^{2}} \mathrm{Z}(\mathrm{x}, \mathrm{t})\right|_{\mathrm{x}=1}=\mathrm{V}(\mathrm{t})+\mathrm{L}^{2}(\mathrm{t})-\mathrm{L}(\mathrm{t})
$$

Then:

$$
\left.\frac{\partial^{3}}{\partial x^{2} \partial t} Z(x, t)\right|_{x=1}=V^{\prime}(t)+2 L(t) L^{\prime}(t)-L^{\prime}(t)
$$

So:

$$
\begin{aligned}
& V^{\prime}(t)+2 L(t) L^{\prime}(t)-L^{\prime}(t)=2 \lambda(t) L(t)-2 \mu(t) V(t) \\
& -2 \mu(t) L^{2}(t)+2 \mu(t) L(t)
\end{aligned}
$$

The differential equation for $\mathrm{L}(\mathrm{t})$ is given in (3) as:

$$
L^{\prime}(t)=-\mu(t) L(t)+\lambda(t)
$$

Therefore:

$$
2 \mathrm{~L}(\mathrm{t}) \mathrm{L}^{\prime}(\mathrm{t})=-2 \mu(\mathrm{t}) \mathrm{L}^{2}(\mathrm{t})+2 \mathrm{~L}(\mathrm{t}) \lambda(\mathrm{t})
$$

This substitution is made on the left side of (5) to yield:

$$
V^{\prime}(t)=\lambda(t)-2 \mu(t) V(t)+\mu(t) L(t)
$$

Subtracting (3) from (6) gives:

$$
V^{\prime}(t)-L^{\prime}(t)=-2 \mu(t) V(t)+2 \mu(t) L(t)
$$

Or:

$$
[\mathrm{V}(\mathrm{t})-\mathrm{L}(\mathrm{t})]^{\prime}=-2 \mu(\mathrm{t})[\mathrm{V}(\mathrm{t})-\mathrm{L}(\mathrm{t})]
$$

Integration gives:

$$
V(t)-L(t)=\left(V_{o}-L_{o}\right) \exp \left(-2 \int_{0}^{t} \mu(s) d s\right)
$$

Finally:

$$
V(t)=L(t)+\left(V_{o}-L_{o}\right) \exp \left(-2 \int_{0}^{t} \mu(s) d s\right)
$$

Substitution from (4) completes the proof. 
A comparison to the $\mathrm{M} / \mathrm{M} / \mathrm{S}$ variance: Rothkopf and Oren (1979) studied some approximations for the mean and the variance of the general M/M/S queuing system. In that research they showed the variance of the distribution of state probabilities to have the differential equation form:

$$
\begin{aligned}
V^{\prime}(t)= & \lambda(t)+\mu(t) S-\mu(t) \sum_{n=0}^{S-1}(2 L(t)+1-2 n) \\
& (S-n) P_{n}(t)
\end{aligned}
$$

$\mathrm{V}(0)=\mathrm{V}_{0}$

In (7), $S$ is the number of servers provided in the system. The terms of the summation in (7) can be rearranged to yield:

$$
\begin{aligned}
& \sum_{n=0}^{\mathrm{s}-1}(2 L(t)+1) S P_{n}(t)-(2 L(t)+1) \\
& n P_{n}(t)-2 n S P_{n}(t)+2 n^{2} P_{n}(t)
\end{aligned}
$$

Now, let $S$ approach $\infty$ and carry out the expectations of the summation. Equation 7 reduces to:

$$
\left.V^{\prime}(t)+2 \mu(t) L^{2}(t)+\mu(t) L(t)-2 \mu(t) L^{2}(t)+V(t)\right)
$$

$$
\mathrm{V}(0)=\mathrm{V}_{0}
$$

Consequently:

$$
\mathrm{V}^{\prime}(\mathrm{t})+2 \mu(\mathrm{t}) \mathrm{V}(\mathrm{t})=\lambda(\mathrm{t})+\mathrm{L}(\mathrm{t}) \mu(\mathrm{t})
$$

This is identical to Eq. 6, the direct result of the derivation from the factorial moment generating function. This new derivation thus serves as a confirmation of the Rothkopf and Oren (1979) variance presentation.

\section{RESULTS}

The state differential-difference equations of the $\mathrm{M} / \mathrm{M} / \infty$ system are given in Eq. 1. If the system is stationary in time then each of the first derivatives in Eq. 1 is equal to zero. The resulting difference equations can be solved sequentially to yield consecutive state probabilities that are Poisson with mean and variance equal to $\lambda / \mu$. The work done here extends that result to the case where the average arrival rate and the average service rate vary over time, being $\lambda(\mathrm{t})$ and $\mu(\mathrm{t})$. The moment generating function is applied to Eq. 1. Summing the results over $\mathrm{n}$ and using $x=1$ in $Z(x, t)$ leads first to a differential equation for the mean $\mathrm{L}(\mathrm{t})$. Further appeal to $\mathrm{Z}(\mathrm{x}, \mathrm{t})$ produces a differential equation for the variance $V(t)$. That differential equation contains $L(t)$ and $L^{\prime}(t)$, so the $V(t)$ differential equation is solved by using the solution found for $\mathrm{L}(\mathrm{t})$. The expressions for $\mathrm{L}(\mathrm{t})$ and $\mathrm{V}(\mathrm{t})$ extend the finding of Rothkopf and Oren (1979). That work presented the variance for the $\mathrm{M} / \mathrm{M} / \mathrm{S}$ system, where $\mathrm{S}$ $<\infty$ is the finite number of servers available.

\section{DISCUSSION}

The results found here show that $\mathrm{L}(\mathrm{t})$ and $\mathrm{V}(\mathrm{t})$ in the $M / M / \infty$ system are related. In fact, $V(t)$ is found because $[\mathrm{V}(\mathrm{t})-\mathrm{L}(\mathrm{t})]^{\prime}=-2 \mu(\mathrm{t})[\mathrm{V}(\mathrm{t})-\mathrm{L}(\mathrm{t})]$. It is seen that if $\mu(t)>0$ and is bounded away from zero, then $V(t)$ $\mathrm{L}(\mathrm{t})$ will approach zero as $\mathrm{t}$ approaches $\infty$. If $\lambda(\mathrm{t})$ and $\mu(\mathrm{t})$ are both constant $\lambda$ and $\mu$ then $\mathrm{L}(\mathrm{t})=\mathrm{L}$ and $\mathrm{V}(\mathrm{t})=$ $\mathrm{V}$ are both equal to $\lambda / \mu$ since the state probabilities are Poisson. It is left as an open research topic to show the nature of $\mathrm{V}(\mathrm{t})-\mathrm{L}(\mathrm{t})$ for all $\mathrm{t}>0$. A particular question is the nature of $\lambda(\mathrm{t})$ and $\mu(\mathrm{t})$ that would result in $\mathrm{L}(\mathrm{t})=$ $\mathrm{V}(\mathrm{t})$ for all $\mathrm{t}>0$.

\section{CONCLUSION}

In the $\mathrm{M} / \mathrm{M} / \infty$ system it is known that the steadystate probabilities are Poisson with parameter $\lambda / \mu$.The mean and variance are thereby equal to each other and to $\lambda / \mu$. The derivation of the general form of the mean and variance of the nonstationary $\mathrm{M} / \mathrm{M} / \infty$ system has been presented here. Two first-order and nonhomogeneous ordinary differential equations are solved sequentially to obtain $\mathrm{L}(\mathrm{t})$ and $\mathrm{V}(\mathrm{t})$. The derivation of the ordinary differential equation for the variance was shown to yield the same result as was obtained from extending the $\mathrm{M} / \mathrm{M} / \mathrm{S}$ variance result from Rothkopf and Oren (1979) to the case of an infinite number of servers.

\section{REFERENCES}

El-Sherbiny, A.A., 2010. Transient solution to an infinite server queue with varying arrival and departure rate. J. Math. Stat., 6: 1-3. http://www.scipub.org/fulltext/jms2/jms2611-3.pdf

Rothkopf, M.H. and S.S. Oren, 1979. A closure approximation for the non-stationary M/M/S queue. Manage. Sci., 25: 522-534. http://www.jstor.org/stable/2630323 